\title{
Susceptibility Distortion Correction for Echo Planar Images with Non-uniform B-Spline Grid Sampling: A Diffusion Tensor Image Study
}

\author{
M.O. Irfanoglu ${ }^{1}$, L. Walker ${ }^{3}$, S. Sammet ${ }^{2}$, \\ C. Pierpaoli ${ }^{3}$, and R. Machiraju ${ }^{1}$ \\ 1 Computer Sciences and Engineering Department, \\ The Ohio State University, USA \\ irfanoglu.1@osu.edu \\ 2 Department of Radiology, \\ The Ohio State University, USA \\ 3 National Institute of Child Health and \\ Human Development, NIH, USA
}

\begin{abstract}
In this paper, we propose a novel method for correcting the geometric distortions in diffusion weighted images (DWI) obtained with echo planar imaging (EPI) protocol. Our EPI distortion correction approach employs a deformable registration framework with the B-splines transformation, where the control point distributions are non-uniform and functions of the expected norm of the spatial distortions. In our framework, the amount of distortions are first computed by estimating the $B_{0}$ fieldmap from an initial segmentation of a distortion-free structural image and tissue susceptibility models. Fieldmap estimates are propagated to obtain expected spatial distortion maps, which are used in the sampling of active B-spline control points. This transformation is flexible in locations with large distortion expectations, yet with relatively few degrees-of-freedom and does not suffer from local optima convergence and hence does not distort anatomically salient locations. Results indicate that with the proposed correction scheme, tensor derived scalar maps and fiber tracts of the same subject computed from data acquired with different phase encoding directions provide better coherency and consistency compared traditional registration based approaches.
\end{abstract}

\section{Introduction}

Diffusion tensor images (DTI) are typically acquired using echo planar imaging (EPI) [1] acquisitions, which are vulnerable to static magnetic field inhomogeneities subsequently leading to image distortions. The amount of distortion in an EPI image is proportional to the static field inhomogeneity. Additionally, images obtained at higher field strengths suffer more from EPI distortion artifacts. The geometric distortions in areas with large magnetic susceptibility gradients, such as the sphenoid sinus, temporal lobe and brain stem can lead to incorrect tensor derived scalar maps and can also result in incorrect fiber tracking. 
EPI distortions and their effects on scalar images is a well investigated problem and several schools of correction algorithms have been proposed. In their pioneering work, Jezzard et al. 2] employed a $B_{0}$ inhomogeneity map (fieldmap), which was computed from two gradient-echo scans with differing echo times. They showed that distortions are significant along the phase-encoding direction and are directly related to the inhomogeneity maps. Several other works followed, which employ the fieldmapping strategy 3. Another approach to the problem involves a non-linear registration of the distorted $B_{0}$ EPI image to a structural anatomically correct MR image [4,5]

Fieldmapping based methods, in spite of their physical intuitiveness, suffer from the difficulty of calculating the phase maps near edges or in regions of high-field inhomogeneity. They also require additional scans and precise measurements, such as dwell time. Additionally 67. show that elastic registration based correction schemes usually outperform fieldmapping approaches. However, elastic registration based approaches usually suffer from the "curse of dimensionality" due to their large parametric space and result in distortion of salient anatomical locations. If the parametric space is reduced to deal with this problem, large distortions at high and ultra-high fields can not be corrected.

In this work, we propose a novel fast and robust algorithm for EPI distortion correction, which combines the strengths of fieldmap approaches and elastic registration. A non-uniform B-spline control grid is constructed, where the image is densely sampled with grid knots at locations with large expected distortions and sparsely sampled at locations where the distortions are homogenous. The expected distortions are obtained by synthesizing an artificial fieldmap based on tissue segmentation maps and tissue susceptibility models. Our methods are physically based due to their relationship to fieldmaps; however, they overcome the shortcomings of fieldmap based techniques given the increased adaptivity. Section 2 describes the main steps of the proposed pipeline, where our fieldmap estimation process is briefly reviewed in Section 2.1 and B-Spline knot sampling is described in Section 2.2. Our experimental setup and validation procedures are described in Section 3 and Section 3.1. The results are presented in Section 4 and the paper is concluded with future directions of Section 5 .

\section{Methodology}

The proposed EPI distortion correction framework is demonstrated in Figure 1. After motion and distortion correction of the DWI data [8], the distortionfree structural $\left(T_{2}\right)$ image is rigidly registered to the specific $b=0 \mathrm{~s} / \mathrm{mm}^{2}$ image. It is then segmented into four classes, white matter, gray matter, cerebrospinal fluid (CSF) and air in the $b=0 \mathrm{~s} / \mathrm{mm}^{2}$ image's native space. The tissue label image is subsequently fed into a fieldmap estimation routine first proposed in [9], later employed in [10]. The estimated fieldmaps are transformed into deformation fields and these deformation fields are used to determine the nonuniform sampling regimen of B-spline knot points. The resulting transformation is used during elastic registration and the final deformation field is applied to each diffusion weighted volume. 


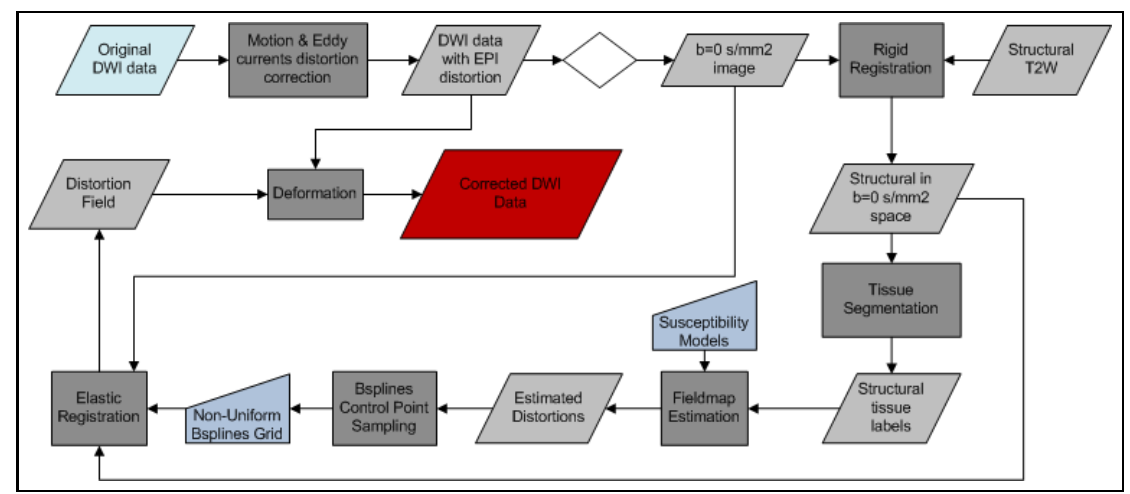

Fig. 1. The flow of the proposed EPI distortion correction framework

\subsection{Fieldmap Estimation}

Our fieldmap estimation process is based on the work of Jenkinson et al. 9], which models the first order perturbations in the main magnetic field $B_{z}^{0}$. Let the susceptibility, $\chi$, be expanded as : $\chi=\chi_{0}+\delta \chi_{1}$, where $\chi_{0}$ is the susceptibility of air $\left(4 \times 10^{-7}\right), \delta$ is the susceptibility difference between tissue and air and $\chi_{1}$ is a binary variable describing the tissue type. The first order perturbations in the magnetic field $B_{z}^{(1)}$ can be written in terms of the main magnetic field $B_{z}^{(0)}$ :

$$
B_{z}^{(1)}=\frac{\chi_{1}}{3+\chi_{0}} B_{z}^{(0)}-\frac{1}{1+\chi_{0}}\left(\left(\frac{\partial^{2} G}{\partial z^{2}}\right) *\left(\chi_{1} B_{z}^{(0)}\right)\right)
$$

where $G$ is the Green's function with $G(x)=(4 \pi r)^{-1}$ with $\left(r=\sqrt{x^{2}+y^{2}+z^{2}}\right)$. The solution to the convolution operation for a single voxel of resolution $(a, b, c)$ :

$$
H(x)=\left(\frac{\partial^{2} G}{\partial z^{2}}\right) *\left(\chi_{1} B_{z}^{(0)}\right)=\sum_{i, j, k \in\{-1,1\}}(i j k) F(x+i a / 2, y+j b / 2, z+k c / 2)
$$

where $F(x)=\frac{1}{4 \pi} \operatorname{atan}\left(\frac{x y}{z r}\right)$. For a set of voxels, due to the linearity of convolution operation, the perturbation field becomes: $B_{z}^{(1)}(x)=\sum_{x^{\prime}} \chi_{1}\left(x^{\prime}\right) H\left(x-x^{\prime}\right)$

Assuming the phase encoding direction along the $y$ axis, the deformation due to this fieldmap can be obtained with:

$$
\Delta y=\frac{\gamma B_{z}^{(1)}(x) N_{y} t_{d w e l l}}{2 \pi}
$$

where $\gamma$ is the gyromagnetic ratio, $\Delta y$ is the pixel shift, $N_{y}$ is the number of voxels along the phase encoding direction and $t_{d w e l l}$ is the dwell time. As described in [10, this model does not account for shimming applied around the edges and might not be adequate for direct use in distortion correction. Figure 2 (a) displays a slice from a structural image and (b) the estimated fieldmap. 


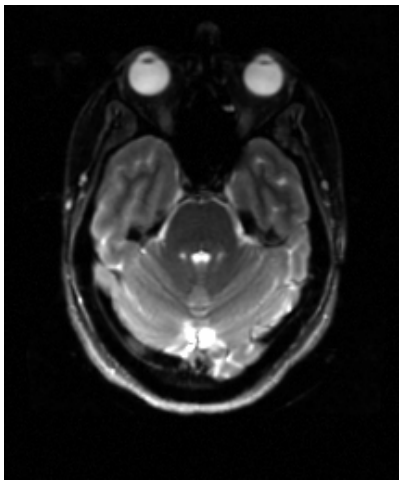

a) Structural image

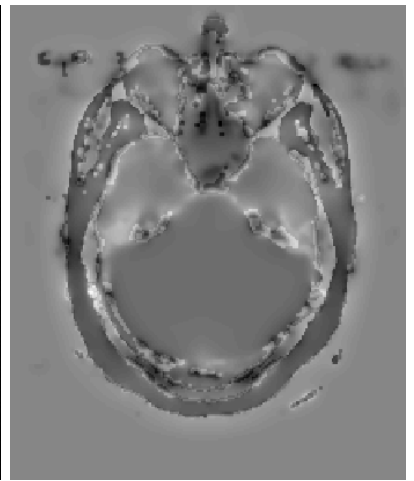

b)Unmasked fieldmap

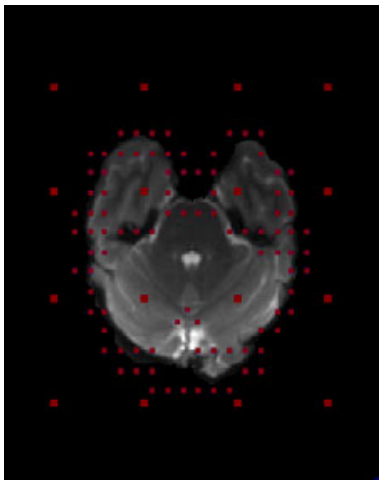

c) Knot point distribution.

Fig. 2. Examples slices for fieldmap estimation from a structural image and the sampling of a B-spline grid points

\subsection{Adaptive B-spline Sampling Using Fieldmaps}

The power of the proposed approach is its ability to model the complex deformations estimated with fieldmaps in a robust manner through a sophisticated physically-based transformation model. This is achieved by nonuniform sampling of B-spline grid locations as a function of the estimated displacements. The sampling of the knot points is performed algorithmically in a multi-resolution fashion as follows: Let $\mathcal{D}_{f}$ be the deformation field estimated using the fieldmaps.

1. Place a B-spline grid of $m \times m \times m$ onto $\mathcal{D}_{f}$. This partitions the image onto $(m-1)^{3}$ cubes. Typically, we set $m=7$.

2. Generate $\mathcal{D}_{e}$ by only using values of $\mathcal{D}_{f}$ on grid locations and interpolating the other voxel displacements using cubic B-Spline kernels.

3. For each cube $\Omega$ :

- Temporarily place a control point $p$ at the center of $\Omega$.

- Recompute $\mathcal{D}_{e}(\Omega)$ for the given cube within the cube $\Omega$.

- If $\left\|\mathcal{D}_{f}(\Omega)-\mathcal{D}_{e}(\Omega)\right\|>\varepsilon$ ( a user-defined threshold),

- Set $p$ as a new control point.

- Replace $\Omega$ with eight new cubes within itself.

- Activate the new cubes.

- Else deactivate $\Omega$.

4. Repeat until no active cubes are present.

This strategy generates a multi-resolution pyramid of B-spline grids, with the initial level containing fully active $7 \times 7 \times 7$ control points (if $m=7$ ), the second level containing partially active $15 \times 15 \times 15$ control points, and so on. Figure 2 (c) displays the knot sampling obtained with this procedure with the displacements computed from the fieldmap in (b). This image is brain masked for clarity purposes. 


\section{$3 \quad$ Experimental Setup}

Five healthy volunteers were scanned on a $3 \mathrm{~T}$ scanner using an eight channel coil. DWI data were acquired with $F O V=24 \times 24 \mathrm{~cm}$, slice thickness $=2.5 \mathrm{~mm}$, matrix size $=128 \times 128,66$ axial slices, parallel imaging factor of 2 . The DWI data set consisted of ten images with $b=0 \mathrm{~s} / \mathrm{mm}^{2}$, ten images with $b=300$ $\mathrm{s} / \mathrm{mm}^{2}$ and 60 images with $b=1100 \mathrm{~s} / \mathrm{mm}^{2}$. For all subjects, two DTI scans were acquired with different phase encode direction (AP and RL) for validation purposes. Structural $T_{2}$ weighted anatomical images $\left(T_{2} W\right)$ were also acquired.

During the elastic registration, the deformation field obtained in the previous level of the pyramid is used to initialize the coefficients of the B-spline at the current level. The optimizer used in the registration is a variant of limited memory Broyden-Fletcher-Goldfarb-Shanno (BFGS) method, which lets the user to set lower and upper bounds for the coefficients. At current level of the pyramid, the active grid points are allowed to move freely, whereas the inactive nodes were constrained to move only within $10 \%$ of the value obtained from the previous level. Additionally, control points were only allowed to move along the phase encoding direction. Up to four levels of the B-spline coefficient pyramid was employed in registration in practice.

\section{$3.1 \quad$ Validation}

For comparison purposes, another elastic registration algorithm with B-spline transformation of uniform grid sampling (e.g., $10 \times 10 \times 10)$ was implemented as reference. The performance of such a registration scheme has been previously shown to outperform that of a fieldmap based approach in most cases [6].

The validation of the proposed correction framework was two-folds: First, the overlap of the structural image, the distorted image, image corrected with the reference algorithm and the image corrected with the proposed algorithm were visually assessed. Second, to quantify the overall improvements in diffusion tensor image quality, probabilistic tractography was carried out on cingulum bundle. The tensor images for every subject, for each phase encode direction and correction scheme, were then fed into a tensor field-based elastic registration routine to compute a population average tensor image and the transformations that mapped each data onto this average brain space. The transformations were subsequently applied to the corresponding tract images in such a way that every single tract image resided in the same coordinate framework. These population connectivity images were first visually analyzed. For these tract images, voxel values were subsequently statistically compared with Wilcoxon Rank test to check for the equality of the medians of number of visitations and to deduce consistency differences between AP and RL; and reference v.s proposed method.

\section{Results}

Figure 3 displays the results for the scalar images. The first column of the figure depicts the original undistorted $T_{2} W$ structural image, the second column 

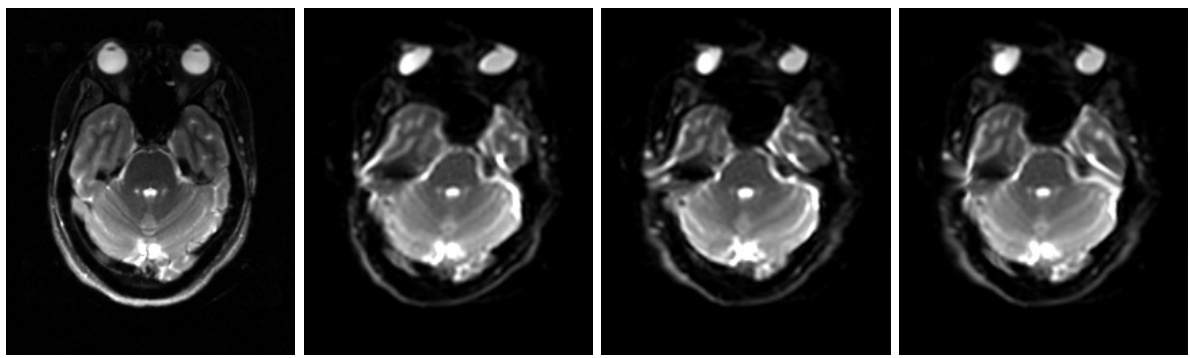

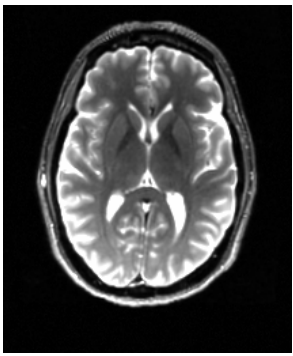

a) Structural image

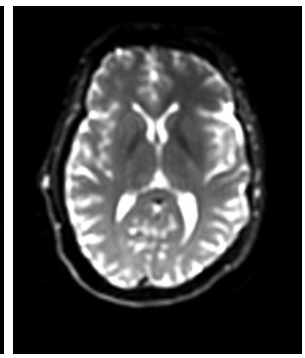

b) Distorted image

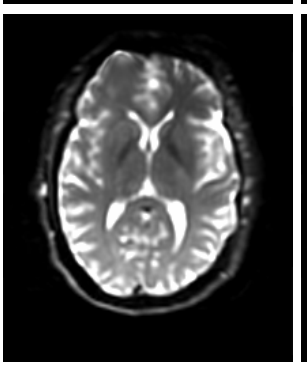

c) Reference

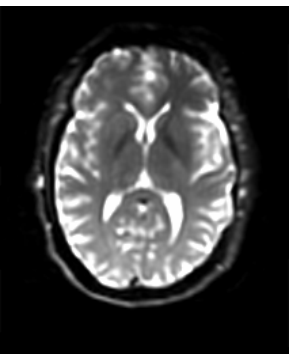

d) Proposed

Fig. 3. Results of the proposed correction algorithm and the reference elastic registration algorithm on the $b=0 \mathrm{~s} / \mathrm{mm}^{2}$ images. Phase encoding direction is RL.

displays slices from the distorted $b=0 \mathrm{~s} / \mathrm{mm}^{2}$ image, third column shows the output of the proposed correction scheme and the last column is for the reference algorithm. The distortion is along the RL direction. In the lower brain, where the distortion is significant, the proposed algorithm performs significantly better than the reference method in the right temporal lobe and left limbic lobe. Both approaches can correct for global displacements but the proposed approach can cope with large local displacements due to its transformation model. For mid-level slices (bottom row), both approaches perform well, with the proposed method showing a slightly better performance around middle frontal gyrus (top left portion of images).

\subsection{Tractography Results}

The average population tracts for the cingulum bundle over 5 subjects, for data acquired with RL and AP distortion are displayed in Figure 4 . In this figure, brightness of tracts indicate the probability of reaching the voxel from the seed ROI. Following conclusions can be drawn from the figure:

- Both correction schemes improve the continuity of the tracts indicated by brighter shades along the tracts. Therefore, one can conclude that EPI distortion correction is an often neglected but crucial step in DTI processing.

- Continuities are also improved with the proposed method. 


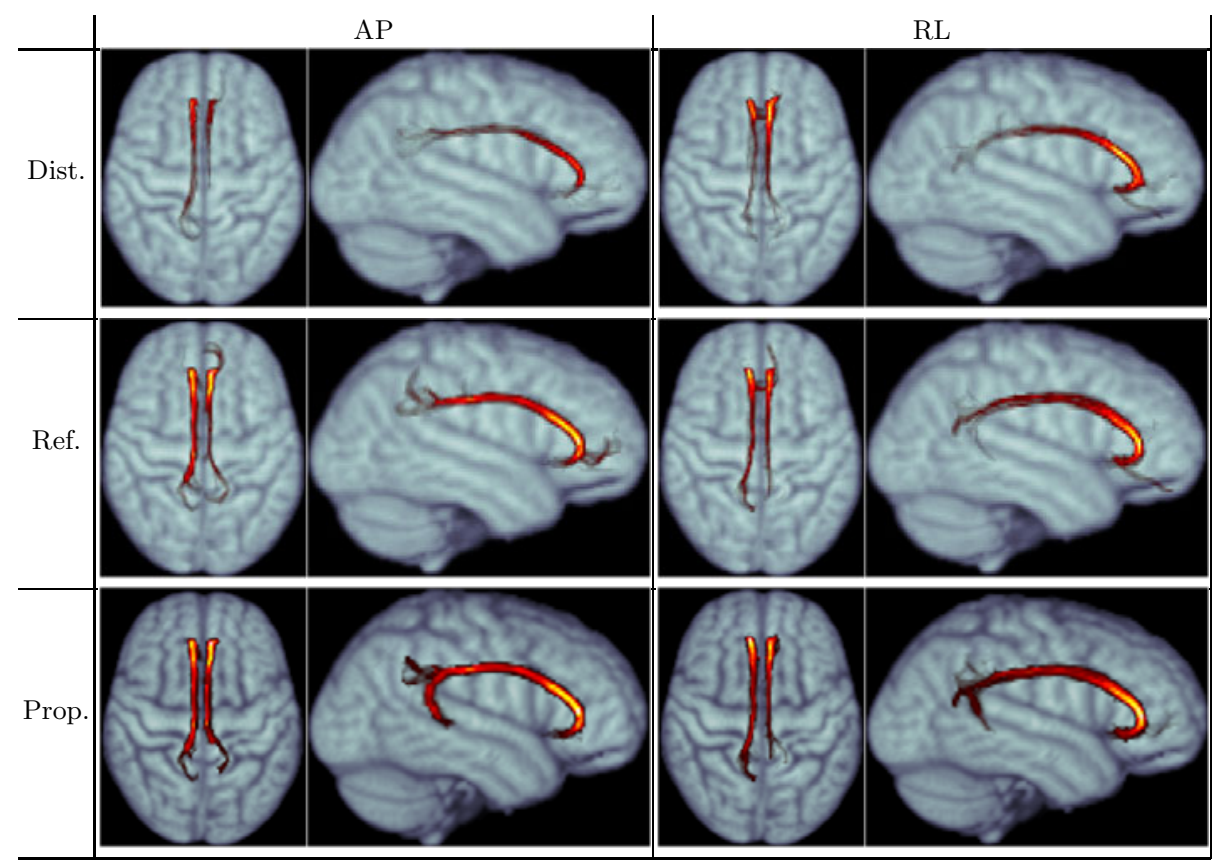

Fig. 4. Population tract averages. Left column displays the results for data acquired with AP distortion and the right column RL distortion. Top row tracts are computed with distorted data, and the bottom row with the proposed correction scheme.

- The proposed method also increases the consistency between data acquired with different distortion directions as the similarity of tracts increases with the reference method compared to the distorted data and with the proposed method compared to the reference approach.

Table 1 displays the results of the statistical tests. These statistics indicate that the proposed correction algorithm results in improved continuity along the tracts. Even though tracts obtained from RL distorted data and AP distorted data are still not statistically significantly similar, a considerable improvement can be observed in their consistency behavior.

Table 1. Statistics on equality of the median of cingulum visitation distributions. The mean values indicate the average number of visitations per voxels along the tracts. 10,000 tracts were initially casted per seed voxel.

\begin{tabular}{|c|c|c|}
\hline$H_{0}$ & $p$-value & $\mu$ \\
\hline$\mu_{R L_{r e f}}=1$ & $1.1910^{-6}$ & \begin{tabular}{|l|l|}
$A P_{\text {ref }}$ & 4275 \\
\end{tabular} \\
\hline$\| \mu_{R L_{p r o p}}=$ & $8.3710^{-3}$ & $R L_{\text {ref }} 3415$ \\
\hline$\mu_{R L_{p r o p}}=\mu_{R L_{\text {ref }}}$ & $1.811^{-7} \mid$ & $\left|\begin{array}{|l|l|}A P_{\text {prop }} & 4776 \\
B L & 3854\end{array}\right|$ \\
\hline$\| \mu_{A P_{\text {prop }}}=$ & & ||$R L_{\text {prop }}|3854|$ \\
\hline
\end{tabular}




\section{Conclusions and Future Work}

In this work, we proposed a novel EPI distortion correction approach that combines the strengths of fieldmap based and elastic registration based correction approaches and minimizes their pitfalls. The algorithm has been shown to perform better than a typical elastic registration based approach both in terms of overlaps of single images and tracts computed from a set of diffusion weighted images of a population. As a future direction, the deformation maps generated by this approach will be used in a point spread function scheme and the effects of EPI signal washout at ultra-high fields will be investigated.

\section{References}

1. Mansfield, P.: Multi-planar image formation using NMR spin echoes. J. Phys. C. 10, $55(1977)$

2. Jezzard, P., Balban, R.: Correction for geometric distortion in echo planar images from b0 field variations. Magnetic Resonance in Medicine 34, 65-73 (1995)

3. Reber, P.J., Wong, E., Buxton, R., Frank, L.R.: Correction of off resonance related distortion in echo-planar imaging using epi-based field maps. Magn. Res. in Med. 39, 328-330 (1998)

4. Kybic, J., Thevenaz, P., Nirkko, A., Unser, M.: Unwarping of unidirectionally distorted EPI images. IEEE Trans. on Medical Imaging 19(2), 80-93 (2000)

5. Ardekani, S., Sinha, U.: Geometric distortion correction of high-resolution 3T diffusion tensor brain images. Magn. Res. in Med. 54, 1163-1171 (2005)

6. Wu, M., Chang, L.C., Walker, L., Lemaitre, H., Barnett, A.S., Marenco, S., Pierpaoli, C.: Comparison of EPI distortion correction methods in diffusion tensor MRI using a novel framework. In: Metaxas, D., Axel, L., Fichtinger, G., Székely, G. (eds.) MICCAI 2008, Part II. LNCS, vol. 5242, pp. 321-329. Springer, Heidelberg (2008)

7. Tao, R., Fletcher, P.T., Gerber, S., Whitaker, R.T.: A variational image-based approach to the correction of susceptibility artifacts in the alignment of diffusion weighted and structural MRI. Inf. Process Med. Imaging 21, 664-675 (2009)

8. Rohde, G., Barnet, A., Basser, P., Marenco, S., Pierpaoli, C.: Comprehensive approach for motion and distortion correction in diffusion weighted MRI. Magnetic Resonance in Medicine 51, 103-114 (2004)

9. Jenkinson, M., Wilson, J.L., Jezzard, P.: Perturbation method for magnetic field calculations of nonconductive objects. Magn. Res. in Med. 52, 471-477 (2004)

10. Poynton, C., Jenkinson, M., Whalen, S., Golby, A.J., Wells, W.: Fieldmap-free retrospective registration and distortion correction for epi-based functional imaging. Med. Image Comput. Comput. Assist Interv. 11, 271-279 (2008) 